\title{
Beneficial effects of $n-3$ polyunsaturated fatty acids on adiponectin levels and AdipoR gene expression in patients with type 2 diabetes mellitus: a randomized, placebo-controlled, double-blind clinical trial
}

\author{
Maryam Mazaherioun ${ }^{1}$, Ahmad Saedisomeolia ${ }^{1,2}$, Mohammad Hassan Javanbakht ${ }^{1}$, \\ Fariba Koohdani ${ }^{1}$, Mohammad Reza Eshraghian ${ }^{3}$, Mahmoud Djalali ${ }^{1}$
}

\begin{abstract}
1Department of Cellular and Molecular Nutrition, School of Nutritional Sciences and Dietetics, International Campus, Tehran University of Medical Sciences, Tehran, Iran ${ }^{2}$ Discipline of Nutrition and Metabolism, School of Molecular Bioscience, University of Sydney, NSW, Sydney, Australia

3Department of Biostatistics, School of Public Health, Tehran University of Medical Sciences, Tehran, Iran
\end{abstract}

Submitted: 23 March 2016

Accepted: 27 June 2016

Arch Med Sci 2017; 13, 4: 716-724

DOI: https://doi.org/10.5114/aoms.2016.62139

Copyright (c) 2016 Termedia \& Banach

\section{Abstract}

Introduction: There is evidence that $n-3$ polyunsaturated fatty acids ( $n-3$ PUFAs) exert beneficial effects to improve type 2 diabetes mellitus (T2DM), but its complications remain poorly understood. Hypoadiponectinemia is one of the important mechanisms responsible for T2DM which necessitates developing novel therapeutic strategies. We aimed to determine the effect of n-3 PUFA supplementation on circulating adiponectin and mRNA expression of adiponectin receptors (AdipoR1, AdipoR2) and Sirt-1 in T2DM patients.

Material and methods: A randomized, double-blind, placebo-controlled trial of 10-week follow-up of $n-3$ PUFAs ( $2.7 \mathrm{~g} /$ day) vs. placebo in T2DM patients $(n=88)$ was conducted. In detail, T2DM patients $(n=44)$ were treated with n-3 PUFAs and the remainder received placebo. Anthropometric and metabolic characteristics were assessed in all participants. Circulating level of adiponectin and mRNA expression of AdipoR1, AdipoR2 and Sirt-1 were measured in peripheral blood mononuclear cells (PBMC) using real-time polymerase chain reaction before and after the intervention.

Results: It was found that n-3 PUFAs increased AdipoR1 gene expression (fold change $=1.321$ in n-3 PUFAs vs. 1.037 in placebo) and AdipoR2 mRNA (fold change $=1.338$ in $n-3$ PUFAs vs. 1.034 in placebo). No significant changes were observed for Sirt-1 expression. The serum level of adiponectin significantly $(p=0.035)$ increased in $n-3$ PUFAs $(5.09$ to $5.58 \mu \mathrm{g} / \mathrm{ml})$ but remained unchanged in the placebo group.

Conclusions: Daily supplementation with n-3 PUFAs (2.7 g) was effective to significantly improve gene expression of AdipoR1 and AdipoR2 and the serum level of adiponectin in T2DM patients. Therefore, $n-3$ PUFAs might emerge as an adjuvant for current antidiabetic therapies. However, confirmatory long-term studies are required.

Key words: $\mathrm{n}-3$ poly-unsaturated fatty acids, type 2 diabetes mellitus, randomized controlled trial, adiponectin, Sirt-1, adiponectin receptors, peripheral blood mononuclear cells.

\author{
Corresponding author: \\ Prof. Mahmoud Djalali \\ Department of Cellular \\ and Molecular Nutrition \\ School of Nutritional \\ Sciences and Dietetics \\ International Campus \\ Tehran University \\ of Medical Sciences \\ HojatDoost St \\ 14155/6117 Tehran, Iran \\ Phone: +98 (21) 88955979 \\ Fax: +98 (21) 88955979 \\ E-mail:mjalali87@yahoo.com
}




\section{Introduction}

Adiponectin, a hormone secreted by adipocytes, plays anti-diabetic and anti-atherogenic roles $[1,2]$. Higher adiponectin levels are attributed to a lower risk of developing type 2 diabetes mellitus (T2DM) with a dose-response relationship $[3,4]$. Glucose-lowering and insulin sensitizing effects have been observed after administration of adiponectin in mice $[2,5,6]$. Augmentation of fatty acid oxidation through activation of AMP kinase $[7,8]$ and peroxisome proliferator-activated receptor (PPAR)- $\alpha$ [9-11] has been shown to explain the insulin-sensitizing effects of adiponectin. These beneficial anti-diabetic effects of adiponectin are operated by adiponectin receptors 1 and 2 (AdipoR1 and AdipoR2), which are expressed in various tissues [12]. Because of the antidiabetic and antiatherogenic effects of adiponectin [5, $6,13,14]$, therapeutic approaches targeting the adiponectin-mediated insulin signaling are ongoing (e.g. thiazolidinedione). Adiponectin activates AdipoR1 and AdipoR2, which alter AMPK and PPAR, respectively, and promote glucose/lipid metabolism, which leads to their anti-diabetic properties being exerted [15]. A pathway from circulating adiponectin to its receptors and AMP kinase to intracellular silent information regulators (SIR) (sirtuins) has been suggested to affect insulin sensitivity and inflammation [2].

Dietary interventions play an important role in therapeutic strategies for patients with T2DM [16]. The $n-3$ series (omega-3) of long-chain polyunsaturated fatty acids (FA), including eicosapentaenoic acid (EPA) and docosahexaenoic acid (DHA), have been demonstrated to play hypolipidemic and antiinflammatory roles, which lead to improvement of metabolism [17]. There is also accumulating evidence supporting the anti-inflammatory and beneficial roles of n-3 PUFAs [18, 19], which might be potential targets for improving hypoadiponectinemia and insulin resistance in T2DM patients.

To date, no randomized, placebo-controlled clinical trial has been conducted to evaluate the effectiveness of $n-3$ PUFA supplementation in patients with T2DM with regard to adiponectin/ AdipoR and Sirt-1 expression. Since the regulation of the expression of AdipoR1 and AdipoR2 in T2DM is poorly understood, we aimed to evaluate the effectiveness of n-3 PUFAs on circulating levels of adiponectin and mRNA expression of AdipoR1, AdipoR2 and Sirt-1.

\section{Material and methods}

\section{Participants and study design}

Eighty-eight T2DM patients were randomly recruited to this double-blind placebo-controlled clinical trial. Patients were diagnosed by an en- docrinologist based on the criteria of the American Diabetes Association with fasting blood sugar (FBS) concentrations higher than $126 \mathrm{mg} / \mathrm{dl}$ (confirmed by testing twice without using antiglycemic medications) and were recruited from the Iranian Diabetes Society. Inclusion criteria was defined as follows: men aged 30-65 years old and premenopausal women older than 30 years, willing to participate, having body mass index (BMI) of 25 to $40 \mathrm{~kg} / \mathrm{m}^{2}$, receiving no dietary supplements, vitamins or herbal products at least 3 months before and throughout the trial and willing to maintain their current diet, physical activity and life style during the 10 weeks of the study.

The exclusion criteria were: receiving insulin or thiazolidinediones medications, having clinical diseases (e.g., kidney, liver, gastrointestinal diseases, type 1 diabetes, retinopathy, neuropathy, microalbuminuria, thyroid and hematological disorders, and malignancies), pregnancy or lactation, having a history of allergic reaction to fish or fish oil, consuming n-3 fatty acid supplements within the last 3 months, receiving anti-obesity drugs such as orlistat or sibutramine, any changes in the type or dosage of medications during the study, and lack of adherence to the trial based on weekly follow-up calls defined as refusing to consume at least $10 \%$ of recommended treatments.

The Ethical Committee of Tehran University of Medical Sciences approved the study procedure (reference number: 25367). All participants completed and signed an informed consent form. The trial was registered at ClinicalTrials.gov (identifier: NCT02261545).

\section{Interventions}

The n-3 PUFA supplement group received 3 soft gels daily for 10 weeks (recommended with meals; each soft gel contained $600 \mathrm{mg}$ EPA and $300 \mathrm{mg}$ DHA; NUTRALAB, Canada, packaged in Zahravi Pharmaceutical Co., Tabriz, Iran). The placebo group received 3 soft gels daily for 10 weeks (recommended to consume with meals; each soft gel contains 900 mg of edible paraffin; provided by Minoo Pharmaceutical, Cosmetic and Hygienic Co., Iran). The placebo soft gel was the same as the supplement in terms of color, shape, taste, and size; both groups received their background therapies. All participants received their own anti-diabetic medications. They were recommended to sustain their diets and physical activity during the trial. Adherence of patients to the interventions was checked by weekly calls and the pill-counting method.

\section{Randomization}

We used permuted block randomization (using Microsoft Excel version 2010 software) for 
allocation of participants to the groups studied, so that T2DM patients $(n=44)$ were treated with $\mathrm{n}-3$ PUFAs and the remainder received placebo A blinded statistician of the project generated sequentially numbered containers for concealment. The blinded secretary of the outpatient diabetes clinic enrolled participants using sealed sequential numbers with attached pockets containing medications (identical placebo and n-3 PUFA supplement soft gels were used). Blinded specialists of the clinic explained the treatments to the participants and checked their adherence. All participants were blind to taking n-3 PUFAs or placebo.

\section{Outcomes and measurements}

Body weight, height, waist circumference and hip circumference were measured according to the standard protocols. Body mass index (BMI) was calculated as body weight $(\mathrm{kg})$ divided by the square of the height $(m)$. After fasting for $12 \mathrm{~h}$, the patients' blood samples were collected before and after the intervention. Blood samples were aliquoted to a test tube and EDTA K3-treated vacutainer in order to perform biochemical analysis and PBMC isolation, respectively. Serum was separated by centrifugation and then was stored at $-80^{\circ} \mathrm{C}$. Fasting blood sugar levels were measured by an Autoanalyzer (Glucose kit Pars Azmoon $\mathrm{Co}$, Iran). $\mathrm{HbA}_{1 \mathrm{c}}$ percentages were measured in whole blood by the immunoturbidimetric method (Cobas Integra 400 plus, Roche Diagnostics, Basel, Switzerland). Serum adiponectin was measured using ELISA Kits (Mediagnost, Germany). Serum insulin was measured using an ELISA kit (Diametre, Italy).

For RNA extraction, PBMC were isolated from anticoagulant-treated blood by standard Ficoll Hypaque (Sigma-Aldrich, USA) density-gradient centrifugation and then RNA was extracted using a Total RNA Extraction Miniprep kit (Qiagen, Germany) according to the manufacturer's protocol. The quality of extracted RNA was checked and the purity was confirmed by spectrophotometry (NanoDrop, Thermo Scientific). cDNA was produced by

Table I. Primers used for this study

\begin{tabular}{|c|}
\hline SIRT-1: Forward GCCGGAAACAATACCTCCAC \\
\hline SIRT-1: Reverse ACACCCCAGCTCCAGTTAG \\
\hline AdipoR1: Forward GGCTGTACTGATGACACCCTT \\
\hline AdipoR1: Reverse GACTCTTCCTCTCACTTCAGC \\
\hline AdipoR2: Forward GACATCTGGTTTCACTCTCATCA \\
\hline AdipoR2: Reverse GTCATAGTCCCTGGAGACTGG \\
\hline$\beta$-actin: Forward CCTGGCACCCAGCACAATGAAG \\
\hline$\beta$-actin: Reverse CTAAGTCATAGTCCGCCTAGAAG \\
\hline
\end{tabular}

reverse transcription of the RNAs of all the patients using a cDNA synthesis kit (Thermo Scientific Fermentas, USA). Then, mRNA expression was determined by standard real-time quantitative reverse transcription-polymerase chain reaction (RT-PCR), which was performed using StepOne System apparatus (Applied Biosystems, Foster City, Calif., USA) with the SYBR Green method (Takara Bio Inc, Japan). Primers for AdipoR1, AdipoR2 and Sirt-1 are summarized in Table I. All gene expression data were normalized to $\beta$-actin $(\Delta C T)$. The fold changes were calculated by the $2-\Delta \Delta C T$ formula (baseline $\Delta C T$ s divided into post-intervention $\Delta C T s$ ).

\section{Statistical analysis}

Quantitative data are expressed as mean \pm SD. Within-group (before/after intervention) differences were compared using the paired $t$-test. Between-group differences were analyzed using the independent $t$-test. Pearson's correlation test was conducted to evaluate pair-wise correlation of the outcome variables. $P$-values less than 0.05 were considered statistically significant. Data were analyzed using SPSS (version 18; Chicago, IL).

\section{Results}

Two-hundred and three patients with T2DM were recruited for initial assessment of eligibility. Figure 1 shows the participants' course during the study (the recruitment period was from July 2014 to January 2015). Eighty-eight T2DM patients were randomly (1: 1 ratio) allocated to the fish oil (n-3 PUFAs) supplement or placebo group, and were followed for 10 weeks (until April 2015).

The studied T2DM patients had a mean \pm SD age of $50.87 \pm 7.30$ years, and BMI of 29.21 $\pm 3.25 \mathrm{~kg} / \mathrm{m}^{2}$. No significant difference was observed in anthropometric and laboratory measurements between intervention groups (Table II). Overall, 85 T2DM patients (44 in the n-3 PUFA group, and 41 patients in the placebo group) successfully finished the trial (losses to follow-up are explained in Figure 1) and were analyzed.

It was found that n-3 PUFAs increased the gene expression levels of AdipoR1 (fold change increase of 1.321 in n-3 PUFAs vs. 1.037 in placebo; $p=0.008$ ) and AdipoR2 (fold change increase of 1.338 in n-3 FAs vs. 1.034 in placebo; $p=0.020$ ) in PBMC of T2DM patients (Figure 2). However, Sirt-1 gene expression levels remained unchanged after interventions. Moreover, the serum level of adiponectin was significantly increased in the $\mathrm{n}-3$ PUFA group (mean difference: $0.48 \mu \mathrm{g} / \mathrm{ml}$; $p=0.001$ ) as compared with placebo (mean difference: $-0.16 \mu \mathrm{g} / \mathrm{ml} ; p=0.234$ ). Differences of outcome variables between the two groups are shown in Tables II and III. 


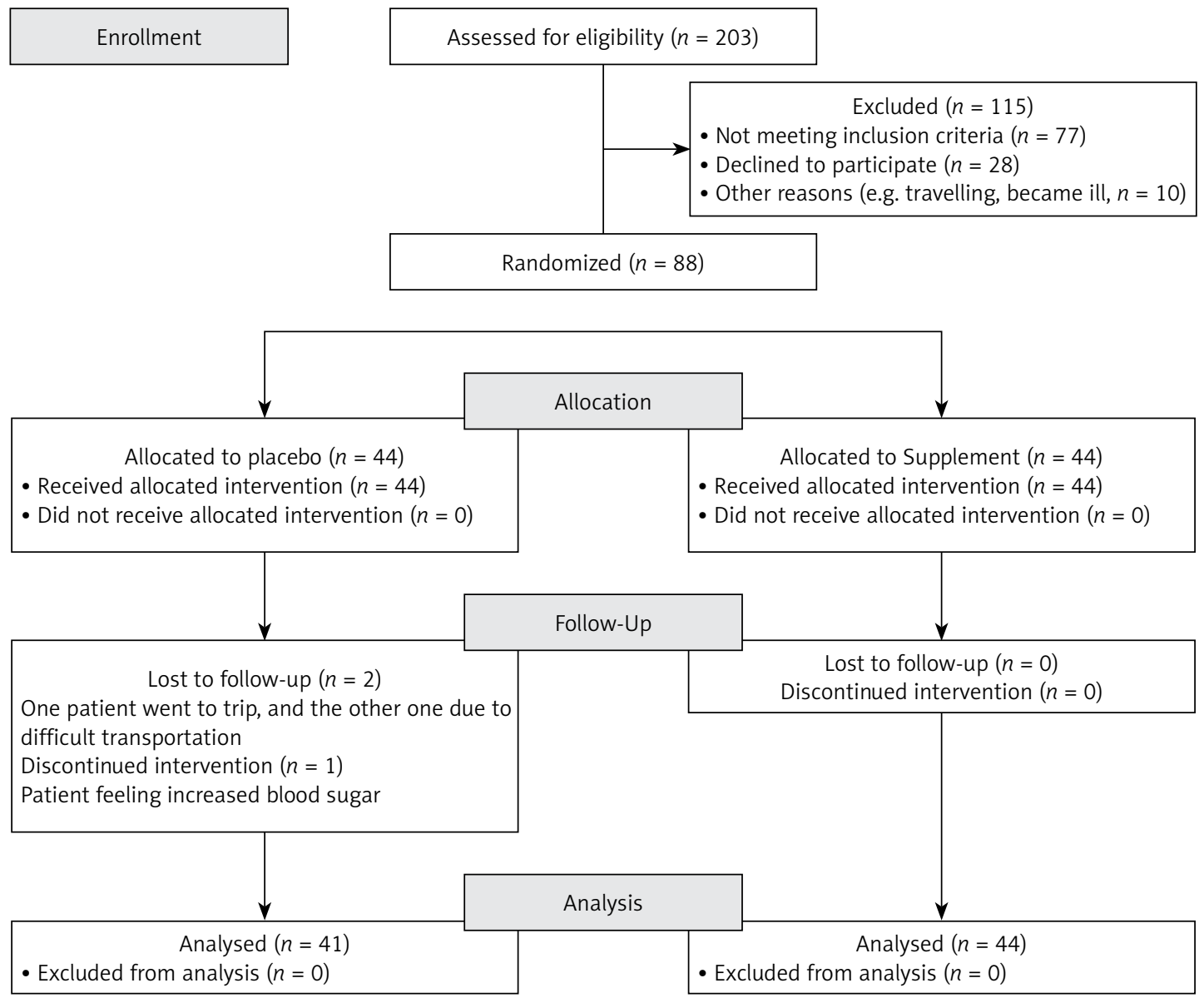

Figure 1. Participants' trial profile during study in the flow diagram

A

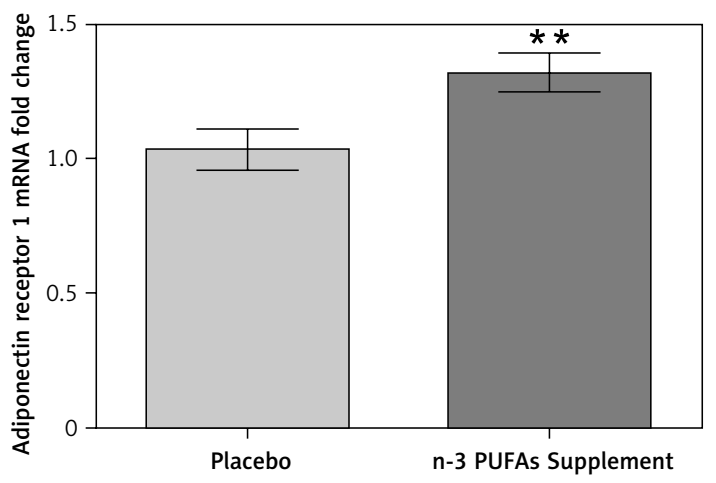

C

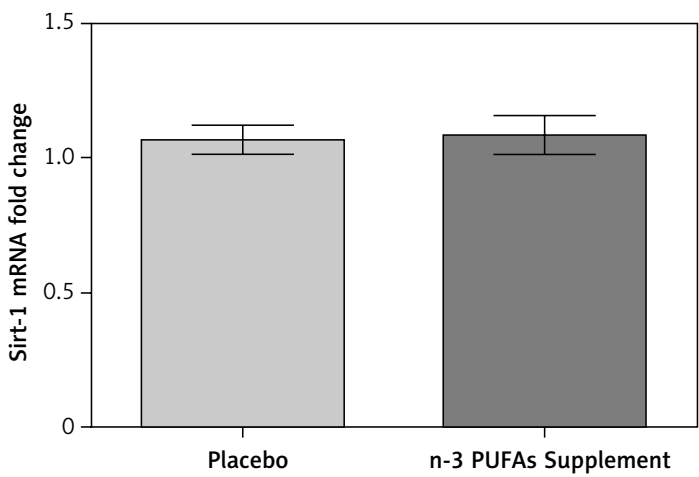

B

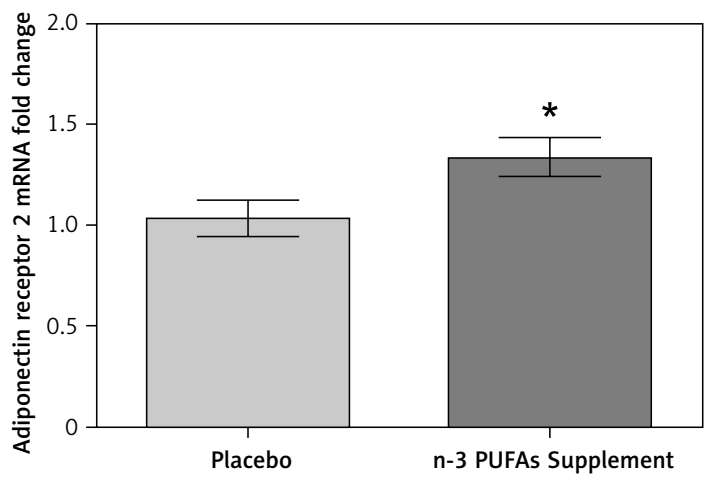

Figure 2. Post-intervention/baseline fold changes of adiponectin receptors (A) AdipoR1, (B) AdipoR2, and (C) Sirt-1 gene expression; in patients with type 2 diabetes mellitus (T2DM) treated with $n-3$ fatty acids ( $n-3$ PUFAs) in comparison with the placebo group

${ }^{*} p<0.05,{ }^{* *} p<0.01$. 
Table II. Baseline characteristics of study participants

\begin{tabular}{|lccc|}
\hline Parameter & Placebo group $(n=41)$ & n-3 group $(n=44)$ & $P_{\text {-value }}^{*}$ \\
\hline Gender (male/female) & $24 / 17$ & $29 / 15$ & 0.483 \\
\hline Age [years] & $50.56(7.21)$ & $51.15(7.45)$ & 0.708 \\
\hline Duration of diabetes [years] & $7.57(2.2)$ & $6.93(1.82)$ & 0.147 \\
\hline Antidiabetic drugs, $n(\%):$ & & \\
\hline Metformin & $23(56 \%)$ & $21(48 \%)$ & 0.44 \\
\hline Metformin + gliclazide & $18(44 \%)$ & $23(52 \%)$ & 0.44 \\
\hline Weight $[\mathrm{kg}]$ & $79.57(12.94)$ & $82.56(15.87)$ & 0.346 \\
\hline Height $[\mathrm{cm}]$ & $1.64(0.09)$ & $1.67(0.102)$ & 0.185 \\
\hline WC $[\mathrm{cm}]$ & $102.49(10.20)$ & $101.72(10.57)$ & 0.734 \\
\hline HIP $[\mathrm{cm}]$ & $106.09(7.39)$ & $106.05(7.92)$ & 0.985 \\
\hline BMI $\left[\mathrm{kg} / \mathrm{m}^{2}\right]$ & $29.21(2.90)$ & $29.22(3.58)$ & 0.991 \\
\hline WHR & $0.96(0.07)$ & $0.95(0.05)$ & 0.564 \\
\hline FBS $[\mathrm{mg} / \mathrm{dl}]$ & $182.26(52.48)$ & $172.11(39.55)$ & 0.315 \\
\hline HbA ${ }_{1 \mathrm{c}}(\%)$ & $7.84(1.12)$ & $7.44(1.08)$ & 0.101 \\
\hline
\end{tabular}

All values are mean (SD). ${ }^{*}$ Significances are based on independent $t$-test, BMI - body mass index, WHR - waist to hip ratio.

Table III. Metabolic characteristics of patients with type 2 diabetes mellitus randomly allocated to placebo and treatment ( $n-3$ fatty acids) interventions

\begin{tabular}{|c|c|c|c|}
\hline Parameter & Placebo group $(n=41)$ & $n-3$ group $(n=44)$ & $P$-value ${ }^{2}$ \\
\hline \multicolumn{4}{|l|}{ FBS [mg/dl]: } \\
\hline Baseline & $182.26(52.48)$ & $172.11(39.55)$ & 0.315 \\
\hline Endpoint & $177.73(48.16)$ & $160.36(42.66)$ & 0.082 \\
\hline$P$-value ${ }^{1}$ & 0.492 & 0.060 & \\
\hline \multicolumn{4}{|c|}{ Insulin [mU/l]: } \\
\hline Baseline & $11.95(4.35)$ & $11.31(5.91)$ & 0.573 \\
\hline Endpoint & $12.30(4.43)$ & $11.54(5.77)$ & 0.504 \\
\hline$P$-value ${ }^{1}$ & 0.105 & 0.337 & \\
\hline \multicolumn{4}{|l|}{$\mathrm{HbA}_{1 \mathrm{c}}(\%):$} \\
\hline Baseline & $7.85(1.13)$ & $7.54(1.11)$ & 0.207 \\
\hline Endpoint & $7.72(1.02)$ & $7.04(0.93)$ & $<0.01$ \\
\hline$P$-value ${ }^{1}$ & 0.36 & $<0.001$ & \\
\hline \multicolumn{4}{|l|}{ QUICKI: } \\
\hline Baseline & $0.304(0.01)$ & $0.311(0.02)$ & 0.15 \\
\hline Endpoint & $0.304(0.01)$ & $0.313(0.02)$ & 0.06 \\
\hline$P$-value ${ }^{1}$ & 0.927 & 0.284 & \\
\hline \multicolumn{4}{|l|}{ HOMA-IR: } \\
\hline Baseline & $5.30(2.08)$ & $4.95(3.30)$ & 0.563 \\
\hline Endpoint & $5.42(2.39)$ & $4.75(3.26)$ & 0.289 \\
\hline$P$-value ${ }^{1}$ & 0.536 & 0.449 & \\
\hline \multicolumn{4}{|c|}{ Adiponectin $[\mu \mathrm{g} / \mathrm{ml}]$ : } \\
\hline Baseline & $4.40(2.62)$ & $5.09(2.79)$ & 0.24 \\
\hline Endpoint & $4.23(2.59)$ & $5.58(3.13)$ & 0.03 \\
\hline$P$-value ${ }^{1}$ & 0.234 & $<0.001$ & \\
\hline
\end{tabular}

${ }^{i}$ Paired-sample t-test. ${ }^{2}$ Independent sample t-test. $P \leq 0.05$ was considered as statistically significant. 
Table IV. Pairwise correlations of serum adiponectin, gene expression of adiponectin receptors (AdipoR) and Sirt-1 (placebo and supplement with n-3 fatty acids)

\begin{tabular}{|lccccccccc|}
\hline Parameter & & $\begin{array}{c}\text { Adiponectin } \\
\text { (baseline) }\end{array}$ & AdipoR1 & AdipoR2 & Sirt-1 & FBS & HOMA-IR & QUICKI & BMI \\
\hline $\begin{array}{l}\text { Adiponectin } \\
\text { (baseline) }\end{array}$ & $r$ & 1 & -0.056 & 0.036 & 0.024 & 0.024 & -0.258 & 0.257 & -0.770 \\
\cline { 2 - 9 } & $p$ & & 0.304 & 0.372 & 0.413 & 0.413 & $<0.01$ & $<0.01$ & $<0.001$ \\
\hline AdipoR1 & $r$ & & 1 & 0.47 & 0.216 & -0.158 & -0.051 & 0.107 & 0.068 \\
\cline { 2 - 9 } & $p$ & & & $<0.001$ & 0.04 & 0.074 & 0.322 & 0.164 & 0.269 \\
\hline AdipoR2 & $r$ & & 1 & 0.578 & -0.120 & -0.104 & 0.180 & -0.017 \\
& $p$ & & & & $<0.001$ & 0.137 & 0.171 & 0.049 & 0.438 \\
\hline Sirt-1 & $r$ & & & 1 & -0.129 & -0.064 & 0.107 & -0.025 \\
& $p$ & & & & & 0.120 & 0.281 & 0.164 & 0.41 \\
\hline
\end{tabular}

HOMA-IR - Homeostasis Model Assessment-Insulin Resistance, QUICKI - Quantitative Insulin Sensitivity Check Index, BMI - body mass index, $r$-Pearson correlation coefficient, $p-p$-value.

An interesting significant pair-wise correlation was found between fold change of gene expression levels of Sirt-1 and adiponectin receptors. There were also significant correlations between the serum adiponectin and HOMA-IR and QUICKI indexes of insulin resistance (Table IV). The serum level of adiponectin and BMI showed a significant inverse association $(r=-0.77 ; p<0.001)$.

To explore the pathway from Sirt-1 to adiponectin receptors, we report a significant correlation between gene expression levels of Sirt-1 and adiponectin receptors (both 1 and 2) (Table IV; $p<0.001)$.

\section{Discussion}

To the best of our knowledge, this study is the first randomized, double-blind, placebo-controlled trial revealing the beneficial effects of supplementation with long chain n-3 PUFAs on serum adiponectin levels and gene expression of its receptors in patients with T2DM.

Type 2 diabetes mellitus has become one of the most important health problems in the world [20], causing micro- and macrovascular complications. Compelling evidence supports the endocrine roles and more complex functions of adipose tissue in insulin resistance, obesity, and T2DM, as adipose tissue releases hormones and cytokines to regulate metabolism and inflammatory pathways [5, $13,21,22]$. There has been abundant interest in adiponectin, which is a versatile regulator of insulin sensitivity, energy homeostasis, inflammation/ atherosclerotic processes, and anti-ischemic cardioprotection mechanisms [6, 13]. Hypoadiponectinemia is closely linked to the pathogenesis of T2DM, since both adipose adiponectin gene expression and serum adiponectin levels have been demonstrated to be significantly reduced in most rodent models of T2DM, and also in humans with type T2DM [5, 6, 13, 23, 24]. The exact mechanism responsible for decreased adiponectin in the obese and T2DM patients is not clearly understood. The increased fat mass results in adipose tissue hypoxia, which has been demonstrated to increase endoplasmic reticular stress. Macrophage infiltration into adipose tissue might lead to a low-grade chronic inflammatory state which is accompanied by increased pro-inflammatory cytokine production [5, 6, 13]. Therapeutic approaches through PPAR- $\gamma$-agonist-mediated adiponectin augmentation (by insulin-sensitizing thiazolidinediones) $[13,25]$ have been widely used. Rosiglitazone has revealed dose-dependent effects on cellular and secreted adiponectin levels in T2DM patients, while metformin had no such effect [26]. The present study showed a slight increase in the circulating level of adiponectin after supplementation of T2DM individuals with n-3 PUFAs, which agrees with the findings of Nomura et al. indicating a significant increase in secreted adiponectin by T2DM patients after EPA therapy [27].

It has been demonstrated that AdipoR1 and AdipoR2 are the functional receptors of adiponectin, increasing AMPK, PPAR- $\alpha$ ligand activity, fatty acid oxidation, and glucose uptake by adiponectin [28]. Nevertheless, there are insufficient human studies supporting the functional roles of adiponectin receptors in the beneficial effects of adiponectin-mediated therapeutic strategies. Li et al. observed that administration of pioglitazone did not alter expression of adiponectin receptors in muscle, adipose tissue, or cellular adipose fractions of humans. They also revealed that baseline or pioglitazone-mediated insulin sensitivity was not correlated with receptor expression [12]. They concluded that adiponectin-mediated beneficial effects of pioglitazone are not necessarily linked to changes of those receptors. This lack of 
correlation between AdipoR and the insulin resistance index is in line with our findings; however, we observed that AdipoR levels are significantly increased by $n-3$ PUFAs. This is in line with the findings of Ramezani et al. [29] which showed that administration of n-3 PUFAs with or without vitamin E for 2 months could significantly increase the gene expression of AdipoR. Regarding the roles of adiponectin receptors in the pathogenesis of T2DM, Weigert et al. reported that AdipoR1 and AdipoR2 proteins were lower in monocytes of T2DM patients when compared with normal-weight individuals; however, mRNA expression levels of these receptors were elevated, possibly due to a feedback mechanism [30]. On the other hand, Wang et al. reported that AdipoR1 mRNA levels were significantly lower in transformed lymphocytes of diabetic African-American patients compared with controls [31]. The lack of correlations between monocytic AdipoR mRNA and serum adiponectin [30], which is similar to our findings, indicates that the circulating level of adiponectin might not be a major regulator of monocytic adiponectin receptor mRNA levels. The alterations of AdipoR and Sirt-1 expression with respect to various antidiabetic therapies need to be explained by further studies. There is a growing body of evidence supporting the roles of Sirt- 1 in glycemic control and insulin sensitivity [32]. Sirt-1 is demonstrated to be involved in insulin secretion regulation, pancreatic $\beta$-cells protection, adiponectin secretion, and improvements of insulin sensitivity through the modifications of post-insulin receptor signaling [32-34]. In one study on 43 healthy men and women (no history of diabetes or cardiovascular disease), Sirt-1 gene expression in peripheral blood mononuclear cell (PBMC) was negatively correlated with homeostatic model assessment-insulin resistance (HOMA-IR), body mass index (BMI), waist circumference, and abdominal visceral fat area, but was positively correlated with adiponectin levels [35]. These findings indicating the links between Sirt-1 gene expression and metabolic characteristics of healthy individuals were not in line with the characteristics of T2DM patients. Here, there is no exact explanation for the lack of correlations between Sirt- 1 and clinical characteristics of insulin resistance. However, it might be due to the diabetic background of the patients, which might have altered their Sirt-1 expression. Qiao and Shao found that Sirt-1 regulates adiponectin expression in mouse adipocytes through enhancing the interaction of two transcription factors (Foxo1 and C/ EBP) which form a transcriptional complex at the adiponectin promoter [36]. The cross-regulation of adiponectin and Sirt-1 is demonstrated to be operative in the adiponectin-mediated activation of AMPK (by binding to AdipoR1), leading to the activation of Sirt-1 and consequent deacetylation of peroxisome proliferator-activated receptor $\gamma$ coactivator $1 \alpha(P G C-1 \alpha)$ [2]. Altogether, activating this adiponectin-Sirt-1 pathway might lead to improvement of glucose and lipid metabolism, insulin sensitivity, mitochondrial function, and oxidative stress [2, 32].

To date, antidiabetic therapeutic approaches through inflammatory pathways and potential signaling pathways have received insufficient attention. However, some evidence supports the beneficial metabolic roles of n-3 PUFAs through anti-inflammatory cascades $[18,19]$. Xue et al. showed that n-3 PUFAs activate the AMPK/Sirt1 pathway, resulting in deacetylation of NF- $\mathrm{B}$. Actually, n-3 PUFAs conduct their anti-inflammatory roles by negative regulation of macrophage inflammation [19]. Modulation of adipokines by dietary n-3 FAs has also been demonstrated to play a paramount role for improvement of insulin sensitivity in muscles and the liver [17]. n-3 PUFAs have been shown to perform hypolipidemic and anti-inflammatory functions which lead to improvement of various metabolic characteristics [17, 37]. A small, randomized, placebo-controlled clinical trial (27 patients) showed that $1.8 \mathrm{~g}$ of $\mathrm{n}-3$ PUFAs for 8 weeks could reduce adiposity and atherogenic markers with no adverse effect on insulin sensitivity in women with type 2 diabetes [38].

The current study had some limitations. Patients were not followed for longer periods, and also we could not measure the n-3 PUFAs levels in erythrocytes after treatment to verify compliance. It should be noted that AMPK and PPAR are involved in the signaling pathway of AdipoR and Sirt-1; hence their evaluation can provide more information regarding underlying mechanism of $n-3$ PUFA impact on the AdipoR1 and Sirt-1 pathway.

Some of the strengths of this study might be summarized as the high completion rate, the double-blind, randomized, placebo-controlled design, and the sufficient sample size to detect small changes.

In conclusion, this randomized, placebo-controlled, double-blind trial revealed that $n-3$ PUFAs could slightly improve adiponectin/AdipoR and consequent glycemic status. The main outcome variables of this study were serum adiponectin and gene expression of adiponectin receptors and Sirt-1, and the circulating level of adiponectin and gene expression of adiponectin receptors were significantly increased in T2DM patients treated with n-3 PUFA supplements. More long-term studies are necessary to confirm the effectiveness and safety of n-3 PUFA supplementation in management of T2DM patients as an adjuvant to current therapies. 


\section{Acknowledgments}

The authors acknowledge the financial support received from International Campus of Tehran University of Medical Sciences (TUMS. IC) to complete the study (grant no: 25367). The authors would like to acknowledge the support of all the participants enrolled in this study.

\section{Conflict of interest}

The authors declare no conflict of interest.

\section{References}

1. Iwabu M, Miki Okada-Iwabu M, Yamauchi T, Kadowaki T. Adiponectin/adiponectin receptor in disease and aging. npj Aging Mechanisms Dis 2015; 1: 15013.

2. Iwabu M, Yamauchi T, Okada-Iwabu M, et al. Adiponectin and AdipoR1 regulate PGC-1alpha and mitochondria by Ca2+ and AMPK/SIRT1. Nature 2010; 464: 1313-9.

3. Li S, Shin HJ, Ding EL, van Dam RM. Adiponectin levels and risk of type 2 diabetes: a systematic review and meta-analysis. JAMA 2009; 302: 179-88.

4. Dinca M, Serban MC, Sahebkar A, et al. Does vitamin D supplementation alter plasma adipokines concentrations? A systematic review and meta-analysis of randomized controlled trials. Pharmacol Res 2016; 107 : 360-71.

5. Kadowaki T, Yamauchi T, Kubota N, Hara K, Ueki K Tobe K. Adiponectin and adiponectin receptors in insulin resistance, diabetes, and the metabolic syndrome. J Clin Investig 2006; 116: 1784-92.

6. Schulze MB, Rimm EB, Shai I, Rifai N, Hu FB. Relationship between adiponectin and glycemic control, blood lipids, and inflammatory markers in men with type 2 diabetes. Diabetes Care 2004; 27: 1680-7.

7. Tomas E, Tsao TS, Saha AK, et al. Enhanced muscle fat oxidation and glucose transport by ACRP30 globular domain: acetyl-CoA carboxylase inhibition and AMP-activated protein kinase activation. Proc Natl Acad Sci USA 2002; 99: 16309-13.

8. Yamauchi T, Kamon J, Minokoshi Y, et al. Adiponectin stimulates glucose utilization and fatty-acid oxidation by activating AMP-activated protein kinase. Nat Med 2002; 8: 1288-95.

9. Fruebis J, Tsao TS, Javorschi S, et al. Proteolytic cleavage product of 30-kDa adipocyte complement-related protein increases fatty acid oxidation in muscle and causes weight loss in mice. Proc Natl Acad Sci USA 2001; 98 2005-10.

10. Yamauchi T, Kamon J, Waki H, et al. Globular adiponectin protected ob/ob mice from diabetes and ApoE-deficient mice from atherosclerosis. J Biol Chem 2003; 278: 2461-8.

11. Yamauchi T, Kamon J, Waki H, et al. The fat-derived hormone adiponectin reverses insulin resistance associated with both lipoatrophy and obesity. Nat Med 2001; 7: 941-6.

12. Li W, Tonelli J, Kishore P. Insulin-sensitizing effects of thiazolidinediones are not linked to adiponectin receptor expression in human fat or muscle. Am J Physiol Endocrinol Metab 2007; 292: E1301-7.

13. Su H, Lau WB, Ma XL. Hypoadiponectinemia in type $2 \mathrm{di}$ abetes: molecular mechanisms and clinical significance. Clin Exp Pharmacol Physiol 2011; 38: 897-904.
14. Stepien M, Wlazeł RN, Paradowski M, et al. Serum concentrations of adiponectin, leptin, resistin, ghrelin and insulin and their association with obesity indices in obese normo-and hypertensive patients - pilot study. Arch Med Sci 2012; 8: 431-6.

15. Okada-Iwabu M, Iwabu M, Ueki K, Yamauchi T, Kadowaki T. Perspective of small-molecule AdipoR agonist for type 2 diabetes and short life in obesity. Diabetes Metab J 2015; 39: 363-72.

16. Veleba J, Kopecky J Jr, Janovska P, et al. Combined intervention with pioglitazone and $n-3$ fatty acids in metformin-treated type 2 diabetic patients: improvement of lipid metabolism. Nutr Metab 2015; 12: 52.

17. Flachs P, Rossmeisl M, Kopecky J. The effect of n-3 fatty acids on glucose homeostasis and insulin sensitivity. Physiol Res 2014; 63 (Suppl. 1): S93-118.

18. Pu J, Liu P. Fatty acids stimulate glucose uptake by the PI3K/AMPK/AKt and PI3K/ERK1/2 pathways. In: Protein Phosphorylation in Human Health. Huang C (ed.). InTech, Rijeka 2012; 478.

19. Xue B, Yang Z, Wang X, Shi H. Omega-3 polyunsaturated fatty acids antagonize macrophage inflammation via activation of AMPK/SIRT1 pathway. PLoS One 2012; 7: e45990.

20. Islam N, Hossain M, Hafizur RM, et al. Fasting glucose to adiponectin ratio is associated with the development of type 2 diabetes mellitus. J Diabetol 2011; 3: 3.

21. Puglisi MJ, Hasty AH, Saraswathi V. The role of adipose tissue in mediating the beneficial effects of dietary fish oil. J Nutr Biochem 2011; 22: 101-8.

22. Pang TTL. Adiponectin and immune tolerance in type 1 diabetes. Thesis. University of Birmingham 2011.

23. Stepien M, Rosniak-Bak K, Paradowski M. Waist circumference, ghrelin and selected adipose tissue-derived adipokines as predictors of insulin resistance in obese patients: preliminary results. Med Sci Monit 2011; 17: PR13-8.

24. Motawi T, Salman T, Shaker O, Abdelhamid A. Association of polymorphism in adiponectin (+45 T/G) and leptin $(-2548 \mathrm{G} / \mathrm{A})$ genes with type 2 diabetes mellitus in male Egyptians. Arch Med Sci 2015; 11: 937-44.

25. Namvaran F, Azarpira N, Rahimi-Moghaddam P, Dabbaghmanesh MH. Polymorphism of peroxisome proliferator-activated receptor gamma (PPARgamma) Pro12Ala in the Iranian population: relation with insulin resistance and response to treatment with pioglitazone in type 2 diabetes. Eur J Pharmacol 2011; 671: 1-6.

26. Phillips SA, Kung J, Ciaraldi TP, et al. Selective regulation of cellular and secreted multimeric adiponectin by antidiabetic therapies in humans. Am J Physiol Endocrinol Metab 2009; 297: E767-73.

27. Nomura S, Shouzu A, Omoto S, et al. Effects of eicosapentaenoic acid on endothelial cell-derived microparticles, angiopoietins and adiponectin in patients with type 2 diabetes. J Atheroscler Thromb 2009; 16: 83-90.

28. Yamauchi T, Kamon J, Ito Y, et al. Cloning of adiponectin receptors that mediate antidiabetic metabolic effects. Nature 2003; 423: 762-9.

29. Ramezani A, Djazayeri A, Koohdani F, et al. Omega-3 fatty acids/vitamin $\mathrm{E}$ behave synergistically on adiponectin receptor-1 and adiponectin receptor-2 gene expressions in peripheral blood mononuclear cell of coronary artery disease patients. Curr Topics Nutraceut Res 2015; 13: 23-32.

30. Weigert J, Neumeier M, Wanninger J, et al. Reduced response to adiponectin and lower abundance of ad- 
iponectin receptor proteins in type 2 diabetic monocytes. FEBS Lett 2008; 582: 1777-82.

31. Wang $\mathrm{H}$, Zhang $\mathrm{H}$, Jia $\mathrm{Y}$, et al. Adiponectin receptor 1 gene (ADIPOR1) as a candidate for type 2 diabetes and insulin resistance. Diabetes 2004; 53: 2132-6.

32. Kitada M, Koya D. SIRT1 in type 2 diabetes: mechanisms and therapeutic potential. Diabetes Metab J 2013; 37 : 315-25.

33. Liang F, Kume S, Koya D. SIRT1 and insulin resistance. Nature Rev Endocrinol 2009; 5: 367-73.

34. Qiang L, Wang H, Farmer SR. Adiponectin secretion is regulated by SIRT1 and the endoplasmic reticulum oxidoreductase Ero1-Lalpha. Mol Cell Biol 2007; 27: 4698-707.

35. Lee H, Chu SH, Park JY, Park HK, Im JA, Lee JW. Visceral adiposity is associated with SIRT1 expression in peripheral blood mononuclear cells: a pilot study. Endocr J 2013; 60: 1269-73.

36. Qiao L, Shao J. SIRT1 regulates adiponectin gene expression through Foxo1-C/enhancer-binding protein alpha transcriptional complex. J Biol Chem 2006; 281: 39915-24.

37. Gross R, Olfson M, Gameroff MJ, et al. Depression and glycemic control in Hispanic primary care patients with diabetes. J Gen Intern Med 2005; 20: 460-6.

38. Kabir M, Skurnik G, Naour N, et al. Treatment for 2 mo with $n-3$ polyunsaturated fatty acids reduces adiposity and some atherogenic factors but does not improve insulin sensitivity in women with type 2 diabetes: a randomized controlled study. Am J Clin Nutr 2007; 86: 1670-9. 